\title{
INTELIGENCIA EMOCIONAL Y SU POTENCIAL PREVENTIVO DE SÍNTOMAS ANSIOSO-DEPRESIVOS Y ESTRÉS EN ESTUDIANTES DE ENFERMERÍA
}

\author{
EMOTIONAL INTELLIGENCE AND ITS PREVENTIVE POTENTIAL \\ FOR ANXIOUS-DEPRESSIVE SYMPTOMS AND STRESS AMONG \\ NURSING STUDENTS
}

\section{INTELIGENCIA EMOCIONAL E SEU POTENCIAL PREVENTIVO PARA SINTOMAS ANSIOSOS-DEPRESSIVOS E ESTRESSE EM ESTUDANTES DE ENFERMAGEM}

\author{
Rodrigo Ardiles Irarrázabal* \\ RenÉ BARRAZA LÓPEZ** \\ Ivannia Koscina Rojas*** \\ Nicole Espínola Salas ${ }^{* * * *}$
}

\begin{abstract}
RESUMEN
Objetivo: Analizar la relación entre inteligencia emocional y síntomas ansioso- depresivos y estrés en estudiantes de enfermería. Material y Método: Estudio cuantitativo observacional transversal, de alcance correlacional en muestra multicéntrica de 80 estudiantes de enfermería de primer ańo $(\mathrm{IC}=90$; error= 0,5); la inteligencia emocional se evaluó con la escala Trait Meta Mood Scale Number 24 (TMMS-24), y la sintomatología depresiva, ansiosa y estrés con la escala Depression, Anxiety and Stress Scale number 21 (DASS-21); como variables de control se consideraron características sociodemográficas y académicas. El análisis descriptivo fue con medidas de frecuencias, tendencia central relativa y pruebas $t$ de Student; para la correlación entre dimensiones, se aplicó coeficiente de correlación de Pearson. Resultados: Se registraron altos niveles de depresión, ansiedad y estrés; la sintomatología depresiva y el estrés correlacionaron con una baja comprensión $(p<0,01)$ y regulación emocional $(p<0,001)$, esta última también mostró correlaciones moderadas con la ansiedad. Las dimensiones de inteligencia emocional y sintomatología evaluada fueron sensibles a la presencia de mal rendimiento académico $(p<0,05)$, asociándose también a mala calidad del sueño y al escaso tiempo de descanso $(p<0,05)$. Conclusión: Una mayor percepción de habilidades para regular y comprender las emociones se asocia con una
\end{abstract}

*Enfermero, Magíster en Acompañamiento Psicoespiritual, Departamento de Enfermería, Facultad de Ciencias de la Salud, Universidad de Antofagasta, Antofagasta-Chile. Escuela de Enfermería, Facultad de Salud, Universidad Santo Tomás, La Serena, Chile. ORCID: https://orcid.org/0000-0001-7427-7772 Email rodrigo.ardiles@uantof.cl. Autor de correspondencia.

**Psicólogo, Doctor en Psicología, Universidad Santo Tomas, Facultad de Ciencias Sociales y Comunicaciones, Escuela de Psicología, La Serena, Chile. ORCID: https://orcid.org/0000-0003-0569-5000 Email: renejavierbarraza@santotomas.cl

***Enfermera, Licenciada en Enfermería, Escuela de Enfermería, Facultad de Salud, Universidad Santo Tomás, La Serena, Chile. ORCID: https://orcid.org/0000-0001-5830-6932 Email: ikoscinarojas@gmail.com

**** Enfermera, Licenciada en Enfermería, Escuela de Enfermería, Facultad de Salud, Universidad Santo Tomás, La Serena, Chile, ORCID: https://orcid.org/0000-0003-1940-258X Email: niicole.espinola@gmail.com 
menor sintomatología de depresión, ansiedad y estrés. Es importante incorporar el desarrollo de la inteligencia emocional en los planes de estudio, a fin de mejorar las condiciones de salud mental de los estudiantes y promover desde la formación una cultura de profesionales empáticos y centrados en la gestión de los cuidados.

Palabras clave: Inteligencia emocional; Ansiedad; Depresión; Estudiantes de Enfermería; Salud mental.

\begin{abstract}
Objective: To analyze the relationship between emotional intelligence and anxious-depressive and stress symptoms in nursing students. Material and Method: Cross-sectional, observational, quantitative study, with a correlational scope, carried out in a multicenter sample of 80 first-year nursing students $(\mathrm{CI}=90$; error= 0.5); emotional intelligence was evaluated with the Trait Meta-Mood Scale Number 24 (TMMS-24), and depressive, anxious and stress symptoms with the Depression, Anxiety and Stress Scale Number 21 (DASS21). In addition, sociodemographic and academic characteristics were considered as control variables. The descriptive analysis was performed using frequency measures, relative central tendency, Student's $t$ tests, and for the correlation between dimensions, Pearson's correlation coefficient was applied. Results: High levels of depression, anxiety and stress were registered. Depressive symptoms and stress correlated with low understanding $(p<0.01)$ and emotional regulation $(p<0.001)$, with the latter also showing moderate correlations with anxiety. The dimensions of emotional intelligence and symptomatology evaluated were sensitive to the presence of poor academic performance $(p<0.05)$ and were also associated with poor sleep quality and little rest time ( $\mathrm{p}$ $<0.05)$. Conclusion: A greater perception of abilities to regulate and understand emotions is associated with less symptoms of depression, anxiety and stress. Results highlight, therefore, the importance of considering the development of emotional intelligence in the curricula, in order to improve the mental health condition of the students and promote a culture of empathetic professionals focused on patient care management.
\end{abstract}

Key words: Emotional intelligence; Anxiety; Depression; Nursing students; Mental health.

\title{
RESUMO
}

Objetivo: Analisar a relação entre inteligência emocional e sintomas ansioso-depressivos e estresse em estudantes de enfermagem. Material e Método: Estudo quantitativo observacional transversal, de escopo correlacional em uma amostra multicêntrica de 80 estudantes de enfermagem de primeiro ano $(\mathrm{IC}=90$; erro= 0,5), a inteligência emocional foi avaliada com a escala Trait Meta Mood Scale Number 24 (TMMS-24), e os sintomas depressivos, ansiosos e estresse com a escala Depression, Anxiety and Stress Scale number 21 (DASS21); características sociodemográficas e acadêmicas foram consideradas como variáveis de controle. A análise descritiva foi realizada com medidas de frequência, de tendência central relativa e testes $t$ de Student; para a correlaçáo entre dimensões, foi aplicado o coeficiente de correlação de Pearson. Resultados: Foram registrados altos níveis de depressão, ansiedade e estresse; sintomas depressivos e estresse correlacionaram-se com baixo entendimento $(p<0,01)$ e regulação emocional $(p<0,001)$, este último também mostrou correlações moderadas com a ansiedade. As dimensóes de inteligência emocional e sintomatologia avaliada foram sensíveis à presença de baixo desempenho acadêmico $(p<0,05)$, estando também associadas à má qualidade do sono e escasso tempo de descanso $(p<0,05)$. Conclusão: Uma maior percepção das habilidades de regular e compreender as emoçóes está associada a menos sintomas de depressão, ansiedade e estresse. É importante incorporar o desenvolvimento da inteligência emocional nos planos de estudo, a fim de melhorar as condiçóes de saúde mental dos estudantes e promover uma cultura de profissionais empáticos e focados na gestáo dos cuidados, desde a formação.

Palavras-chave: Inteligência emocional; Ansiedade; Depressão; Estudantes de enfermagem; Saúde mental.

Fecha de recepción: 7/04/2020

Fecha de aceptación: 15/06/2020 


\section{INTRODUCCIÓN}

La depresión está clasificada por la Organización Mundial de la Salud como uno de los trastornos de la salud más frecuentes en el mundo y de mayor impacto, afectando a más de 300 millones de personas y constituyendo una de las principales causas de discapacidad ${ }^{(1)}$.

En Chile, el problema es particularmente importante, puesto que actualmente es uno de los países que exhibe las más altas tasas de depresión $y$ de trastornos afectivos a nivel mundial ${ }^{(2,}$ 3); se estima que un tercio de la población chilena ha tenido algún trastorno psiquiátrico durante su vida ${ }^{(4)}$. Esta incidencia es aún más alta en la población universitaria ${ }^{(5)}$, siendo los estudiantes del área de la salud los que alcanzan los índices más altos ${ }^{(6-8)}$. La depresión, ansiedad y estrés constituyen un problema silencioso en estudiantes de la salud, problema que no ha sido atendido en forma adecuada ${ }^{(9)}$, y que podría tener consecuencias muy negativas en el desarrollo de las habilidades profesionales y socioemocionales, que son imprescindibles para un buen desempeño de enfermeros y enfermeras ${ }^{(10)}$. En este mismo sentido, como plantea Barraza et al.(9), la alta prevalencia de depresión, ansiedad y estrés, asociada a estudiantes del área de la salud, especialmente a estudiantes de primer ańo, no solo afectaría negativamente su autopercepción emocional, sino que despotencia los recursos emocionales disponibles para manejar los efectos negativos generados por la sobrecarga académica que impone la carrera, dificultando, según Garg et al. ${ }^{(11)}$, sus procesos de adaptación a la vida universitaria que comienza.

Por otro lado, la inteligencia emocional ${ }^{(12,13)}$ se puede definir como una "capacidad útil para monitorear los sentimientos propios y de las otras personas, permitiendo discriminar y utilizar la información que entregan las emociones para guiar el pensamiento y las acciones que posibilitan una interacción social eficaz". A su vez, la autopercepción de la inteligencia emocional corresponde a la capacidad consciente de percibir, comprender y regular las emociones propias, controlando las emociones negativas y propiciando las positivas ${ }^{(7,14-}$ 16). Algunas investigaciones refieren que, cuando los estudiantes de salud exhiben adecuada percepción de su propia inteligencia emocional, esta se asocia a una percepción de menor estrés, ansiedad y depresión, actuando como un factor protector de la carga emocional negativa ${ }^{(5,17)}$ y potenciando ámbitos de actuación como: la toma de decisiones clínicas $^{(18)}$, las competencias quirúrgicas ${ }^{(19)}$, y el trabajo en base a un enfoque más empático ${ }^{(20)}$.

En este sentido, la interacción social es una habilidad de gran importancia en la formación de estudiantes de enfermería, puesto que las habilidades socio-emocionales son fundamentales para un buen desarrollo profesional y para el cuidado de los pacientes ${ }^{(20,21)}$; este cuidado, a su vez, constituye la esencia del desempeńo profesional de un/a enfermero/a. Otro aspecto clave de la interacción social es el desarrollo de la inteligencia emocional, la cual mejora las destrezas interpersonales ${ }^{(1)}$, la comunicación, el razonamiento clínico, el pensamiento crítico, la relación con los pacientes ${ }^{13,}$ 22) y controla estados emocionales negativos que comúnmente se dan en el desempeño profesional en el área de salud ${ }^{(23)}$.

Dados los múltiples beneficios que entrega el desarrollo de la inteligencia emocional ${ }^{(24)}$ y los resultados arrojados por las investigaciones previas se ha hipotetizado que incentivar el desarrollo de la misma serviría como mecanismo de prevención y control de los síntomas depresivos, ansiosos y estrés en estudiantes de salud ${ }^{(9,25)}$

En este contexto, la evidencia a nivel internacional respecto a la inteligencia emocional en estudiantes de enfermería es muy escasa, posiblemente a lo reciente y poco estudiado del tema en este grupo específico. Según lo anterior, el objetivo del presente estudio fue cuantificar la relación entre el nivel de inteligencia emocional autorreportada, con la presencia de sintomatología depresiva, ansiosa y estrés, en una muestra de estudiantes de enfermería de primer año.

\section{MATERIAL Y MÉTODO}

Tipo de estudio y unidad de análisis: La investigación se desarrolló desde un enfoque cuantitativo, bajo un diseño observacional de corte retrospectivo, de carácter transversal y de alcance correlacional. Participaron estudiantes universitarios de enfermería que se encontraban cursando su primer ańo, pertenecientes a dos universidades chilenas.

Muestra: La población de estudiantes de enferme- 
ría de primer año de ambas universidades fue de 112; se seleccionaron estudiantes de este nivel de carrera para enfocar la investigación en la fase inicial del proceso formativo, en el entendido que es una etapa de particular vulnerabilidad. El tamaño muestral óptimo fue estimado con un intervalo de confianza del $90 \%$, con una varianza de 0,5 y un error de $5 \%$. Del total de convocados a responder las encuestas, 32 rechazaron participar del estudio, por lo tanto, la muestra final, no probabilística, quedó conformada por 80 estudiantes de enfermería (71,4\% del universo) pertenecientes a la cohorte 2017 que firmaron el consentimiento informado. De ellos, 27 pertenecían a una universidad de la ciudad de Antofagasta y 53 a una universidad de la ciudad de La Serena. Dado el diseño de muestreo utilizado, no fue posible establecer una representación a la población en forma homogénea por sexo, ni por centro formativo.

Recolección de datos: Como instrumentos de medición se utilizaron dos escalas de autorreporte: Trait Meta Mood Scale number 24 (TMMS-24) ${ }^{(26)}$, para evaluar la inteligencia emocional, y Depression, Anxiety and Stress Scale number 21 (DASS-21) ${ }^{(27)}$, para evaluar la depresión, ansiedad y estrés.

La escala TMMS-24 mide el nivel de autopercepción de las dimensiones de la inteligencia emocional: percepción, comprensión y regulación. La percepción emocional se entiende como la capacidad para percibir los propios estados emocionales; los rangos de adecuación para hombres están entre los $27 \pm 6$ puntos y en mujeres $30 \pm 6$. La comprensión emocional es entendida como la capacidad para comprender los estados emocionales, cuyos rangos de adecuación están entre los 30,5 $\pm 5,5$ puntos para los hombres y $29 \pm$ 6 en mujeres. Por último, la regulación emocional es definida como la capacidad para regular los estados emocionales negativos, y sus rangos de adecuación para los hombres están entre los 29,5 \pm 6,5 puntos y $29 \pm 6$ en mujeres. Los puntajes bajo estos rangos indican necesidad de mejora en la habilidad, en tanto que los puntajes sobre el rango medio indican un alto nivel de autopercepción en el desarrollo de la habilidad, excepto en la atención emocional, donde puntuaciones altas también indican la necesidad de mejora en dicha habilidad ${ }^{(26)}$.

Para este estudio, en el Nivel de percepción emocional "Adecuado" se consideró a hombres que están entre los $27 \pm 6$ puntos y en mujeres $30 \pm$
6, es decir, en las categorías "adecuada” y "buena” de la prueba; como "Inadecuados" a aquellos fuera de estos rangos y sobre estos, es decir, a los de la categoría "debe mejorar". En el Nivel de comprensión emocional "Adecuado" a los con 30,5 $\pm 5,5$ puntos para hombres y $29 \pm 6$ en mujeres, es decir, en las categorías "adecuada" y "buena"; en tanto que se clasificó como "Inadecuados" a aquellos sujetos con puntajes fuera de estos rangos, por tanto, a aquellos sujetos que calificaron en la categoría "debe mejorar". Finalmente, para el Nivel de regulación emocional se consideró "Adecuado" a aquellos sujetos que sus puntajes están entre los $29,5 \pm 6,5$ puntos para hombres y $29 \pm 6$ en mujeres, esto es, se calificaron en las categorías "adecuada” y "buena" de la prueba; en tanto que se clasificó como "Inadecuados" a aquellos sujetos con puntajes fuera de estos rangos, es decir, a aquellos sujetos que calificaron en la categoría "debe mejorar" (Tabla 1). Los puntajes de corte para cada una de estas dimensiones son: percepción $\geq 21$ (debe mejorar su atención emocional), comprensión $\geq 24$ (debe mejorar la comprensión emocional) y regulación $\geq$ 23 (debe mejorar su regulación emocional).

La escala DASS-21 establece normalidad o severidad de cada una de las patologías autorreportadas, cuyos rangos de severidad para depresión clasifican como "normal" de 0-9 puntos para depresión, 0-7 para ansiedad y 0-14 puntos para estrés, aumentando desde niveles "medios" hasta "severidad extrema" (10 a > 28 para depresión; 8 a $>20$ para ansiedad y 15 a $>37$ para estrés) ${ }^{(27)}$. La escala DASS-21 mide síntomas de depresión, ansiedad y estrés, desde el nivel leve a muy severo. Los puntajes de corte para cada una de estas dimensiones son: depresión $\geq 10$, ansiedad $\geq$ 8 y estrés $\geq 15$. Ambas escalas son de autorreporte (evaluadas por el mismo participante), de tipo Likert (escala con diferentes grados de conformidad) y su uso ha sido validado en la población chilena ${ }^{(26,27)}$ (Tabla 2).

Tanto las dimensiones de la inteligencia emocional, como la sintomatología ansiosa, depresiva y estrés, son consideradas las variables principales del estudio. Adicionalmente se aplicó un tercer cuestionario de variables secundarias de control, que consideró factores demográficos, sociales, académicos, hábitos y antecedentes de salud, creado según la literatura y validado en base a juicio experto; su aplicación tuvo como finalidad 
controlar el sesgo en la selección de la muestra, dado el potencial efecto que, según la literatura ${ }^{(28)}$, pudieran tener estos factores sobre la inteligencia emocional y la depresión de los participantes (Tabla 3).

A los que participaron del estudio se les informó el propósito e importancia de la investigación y el tiempo de duración de los cuestionarios. Las encuestas fueron implementadas en la plataforma Google Form y respondidas en noviembre del año 2017 previo consentimiento informado.

Análisis de datos: El análisis estadístico incluyó la descripción de las variables principales, así como las secundarias de control a través de medidas de tendencia central, desviación y dispersión, también se observó el comportamiento comparado de las variables principales en función de las variables secundarias mediante la prueba $t$ de Student. Finalmente se correlacionó las dimensiones de la inteligencia emocional con depresión, ansiedad y estrés mediante el coeficiente de correlación de Pearson. Para la realización de estos análisis se utilizó el software SPSS versión 20.

El estudio fue evaluado y aprobado por el Comité de Ética de la institución patrocinante (Código: No 68/2017).

\section{RESULTADOS}

Inteligencia emocional: Los resultados de la autopercepción de la inteligencia emocional mostraron que la mayor parte de los estudiantes presentó niveles adecuados en cada una de las dimensiones de esta variable. La dimensión percepción emocional, fue la que exhibió una mayor proporción de casos en rango adecuado $(X=26,3 ; D E=7)$. Las medidas de distribución de cada una de las dimensiones se encontraban dentro de los parámetros de normalidad (Tabla 1).

Sintomatología depresiva, ansiosa y estrés: Cerca de la mitad de los encuestados presentaron síntomas característicos de depresión $(X=11,1 ; D E=$ $8,9)$ y estrés $(X=12,5 ; D E=9,6)$, evidenciando un aumento de la proporción de casos para los síntomas de ansiedad ( $X=17,8 ; D E=9,8)$. Las medidas de distribución de cada una de las dimensiones se encontraban dentro de los parámetros de normalidad (Tabla 2). Cabe mencionar que, se consideraron como sintomáticos a los individuos que se calificaron entre las categorías moderada a muy severa, para cada una de las tres variables.

Tabla 1. Dimensiones de la inteligencia emocional en escala TMMS-24, estudiantes primer ańo enfermería, 2017 $(\mathrm{n}=80)$.

\begin{tabular}{llccccc}
\hline Dimensiones & & Casos & $\mathbf{9}$ & Media & DE & KS \\
\hline Nivel de percepción emocional & Inadecuado & 10 & $12,5 \%$ & 26,3 & 7 & 0,517 \\
& Adecuado & 70 & $87,5 \%$ & & & \\
\hline Nivel de comprensión emocional & Inadecuado & 31 & $39,0 \%$ & 26,4 & 7,4 & 0,511 \\
& Adecuado & 49 & $61,0 \%$ & & & \\
\hline Nivel de regulación emocional & Inadecuado & 25 & $31,0 \%$ & 27,6 & 7,5 & 0,501 \\
& Adecuado & 55 & $69,0 \%$ & & & \\
\hline
\end{tabular}

Tabla 2. Distribución porcentual de sintomatología depresiva, ansiosa y estrés autorreportado por estudiantes de enfermería, escala DASS-21, 2017 ( $\mathrm{n}=80)$.

\begin{tabular}{llccccc}
\hline Síntomas & & Casos & \% & Media & DE & KS \\
\hline Síntomas de depresión & Asintomático & 41 & $51 \%$ & 11,1 & 8,9 & 0,062 \\
& Sintomático & 39 & $49 \%$ & & & \\
\hline Síntomas de ansiedad & Asintomático & 29 & $36 \%$ & 17,8 & 9,8 & 0,470 \\
& Sintomático & 51 & $64 \%$ & & & \\
\hline Síntomas de estrés & Asintomático & 35 & $44 \%$ & 12,5 & 9,6 & 0,136 \\
& Sintomático & 45 & $56 \%$ & & & \\
\hline
\end{tabular}


Tabla 3. Distribución frecuencial y porcentual de variables de control, n= 80, 2017.

\begin{tabular}{|c|c|c|c|c|}
\hline Variables de control & & & $\mathrm{Fr}$ & $\%$ \\
\hline \multirow[t]{4}{*}{ Demográficas } & Sexo & Hombre & 14 & 17,50 \\
\hline & & Mujer & 66 & 82,50 \\
\hline & Estado civil & Union libre & 4 & 5,00 \\
\hline & & Soltero & 76 & 95,00 \\
\hline \multirow[t]{14}{*}{ Sociales } & Hijos & sí & 7 & 8,75 \\
\hline & & no & 73 & 91,25 \\
\hline & Procedencia & Urbano & 75 & 93,75 \\
\hline & & Rural & 5 & 6,25 \\
\hline & Trabaja & sí & 18 & 22,50 \\
\hline & & no & 62 & 77,50 \\
\hline & Problemas familiares & sí & 27 & 33,75 \\
\hline & & no & 53 & 66,25 \\
\hline & Apoyo social & sí & 73 & 91,25 \\
\hline & & no & 7 & 8,75 \\
\hline & Problemas económicos & sí & 33 & 41,25 \\
\hline & & no & 47 & 58,75 \\
\hline & Credo religioso & sí & 52 & 65,00 \\
\hline & & no & 28 & 35,00 \\
\hline \multirow[t]{18}{*}{ Hábitos } & Consumo de alcohol & sí & 46 & 57,50 \\
\hline & & no & 34 & 42,50 \\
\hline & Consumo de tabaco & sí & 13 & 16,25 \\
\hline & & no & 67 & 83,75 \\
\hline & Consumo de drogas & sí & 19 & 23,75 \\
\hline & & no & 61 & 76,25 \\
\hline & Actividad física & sí & 39 & 48,75 \\
\hline & & no & 41 & 51,25 \\
\hline & Alimentación saludable & sí & 31 & 38,75 \\
\hline & & no & 49 & 61,25 \\
\hline & Calidad de sueño & sí & 21 & 26,25 \\
\hline & & no & 59 & 73,75 \\
\hline & Tiempo a solas & sí & 38 & 47,50 \\
\hline & & no & 42 & 52,50 \\
\hline & Poco tiempo de descanso & sí & 54 & 67,50 \\
\hline & & no & 26 & 32,50 \\
\hline & Actividades recreativas & sí & 45 & 56,25 \\
\hline & & no & 35 & 43,75 \\
\hline \multirow[t]{6}{*}{ Académicas } & Rendimiento académico deficiente & sí & 47 & 58,75 \\
\hline & & no & 33 & 41,25 \\
\hline & Carga académica & sí & 67 & 83,75 \\
\hline & & no & 13 & 16,25 \\
\hline & Experiencia universitaria previa & sí & 21 & 26,25 \\
\hline & & no & 59 & 73,75 \\
\hline \multirow[t]{2}{*}{ Antecedentes de salud } & Patología psiquiátrica familiar & sí & 21 & 26,25 \\
\hline & & no & 59 & 73,75 \\
\hline
\end{tabular}


La Tabla 3 muestra el comportamiento de cada una de estas variables de control.

Niveles de depresión, ansiedad y estrés según variables de control: Se realizó la comparación de los puntajes de depresión, ansiedad y estrés, a partir de cada una de las variables de control y se encontró una mayor presencia de síntomas depresivos, entre quienes habían tenido experiencia universitaria previa, respecto de aquellos estudiantes que no la tenían $\left(t_{(78)}=2,26 ; \mathrm{SE}=5,74 ; p<0,05\right)$; también entre quienes presentaban antecedentes de patología psiquiátrica familiar y el grupo que no presentaba $\left(t_{(78)}=-2,70 ; \mathrm{SE}=-4,46 ; p<0,01\right)$; además entre quienes presentaban rendimiento académico deficiente y los que no presentaban $\left(t_{(78)}=3,51 ; \mathrm{SE}=6,72 ; p<0,001\right)$ (Figura 1). Para la ansiedad, se encontró una mayor presencia de síntomas ansiosos en los alumnos con rendimiento académico deficiente y los que no presentaban $\left.t_{(28)}=3,96 ; \mathrm{SE}=2,02 ; p<0,05\right)$, y en aquellos con poco tiempo de descanso $\left(t_{(78)}=2,31 ; \mathrm{SE}=2,25 ; p\right.$ $<0,05$ ) (Figura 2). En cuanto al estrés, se encontró una mayor incidencia en los alumnos que no realizaban actividad física $\left(t_{(77)}=2,06 ; \mathrm{SE}=2,15 ; p\right.$ $<0,05)$, en los que tenían una mala alimentación saludable $\left(t_{(78)}=-2,49 ; \mathrm{SE}=2,18 ; p<0,05\right)$, en los que presentaban un rendimiento académico deficiente $\left(t_{(78)}=4,26 ; \mathrm{SE}=2,02 ; p<0,05\right)$, en los que reportaban tener poco tiempo de descanso $\left(t_{(78)}=\right.$ 3,51; $\mathrm{SE}=2,19 ; p<0,05)$, como también en los que tenían una mala calidad de sueño $\left(t_{(78)}=-2,90 ; \mathrm{SE}=\right.$ $2,38 ; p<0,05)$ (Figura 3). Las restantes variables de control no mostraron diferencias estadísticamente significativas para ansiedad, depresión y estrés.

Niveles de percepción, comprensión y regulación emocional según variables de control: Con relación a las dimensiones de la inteligencia emocional, se procedió a comparar su comportamiento según las variables de control y se encontraron diferencias significativas para la variable percepción emocional entre quienes presentaban rendimiento académico deficiente $\left(t_{(78)}=-2,19 ; \mathrm{SE}=7,399 ; p<0,031\right)$ (Figura 4). Para la variable comprensión emocional, se encontraron diferencias significativas entre los grupos que presentaban rendimiento académico deficiente $\left.t_{(78)}=-3,21 ; \mathrm{SE}=-5,12 ; p<0,05\right)$, entre quienes reportaban realizar y no realizar actividades recreativas $\left(t_{(78)}=-2,98 ; \mathrm{SE}=4,75 ; p<0,01\right)$, entre los grupos que reportaban tener poco tiempo de descanso y adecuado tiempo de descanso $\left(t_{(78)}=\right.$ -2,23; $\mathrm{SE}=-3,85 ; p<0,05)$, como también entre los que reportaban tener buena y mala calidad de sueño $\left(t_{(78)}=2,94 ; \mathrm{SE}=5,23 ; p<0,01\right)$ (Figura 5).

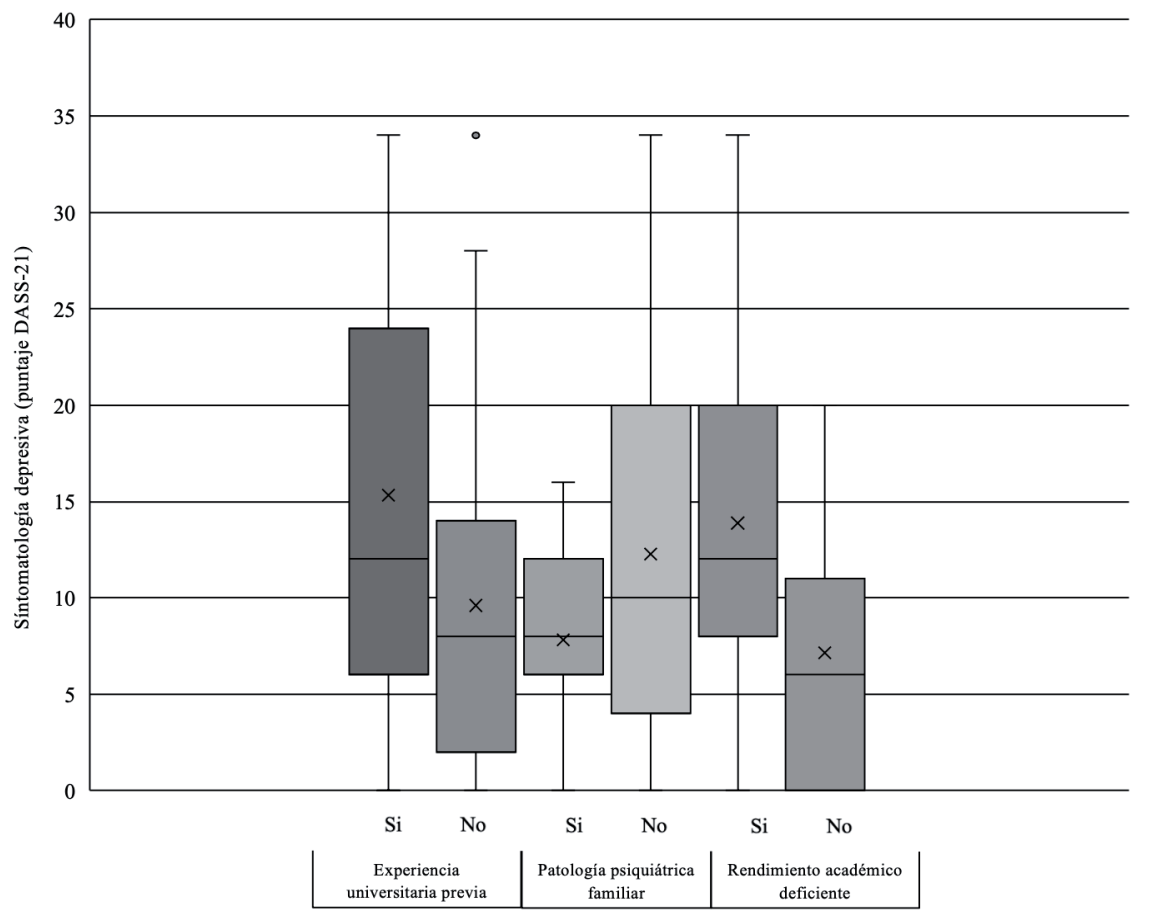

Figura 1. Variables de control que presentan diferencias significativas respecto de la autopercepción de sintomatología depresivos. 


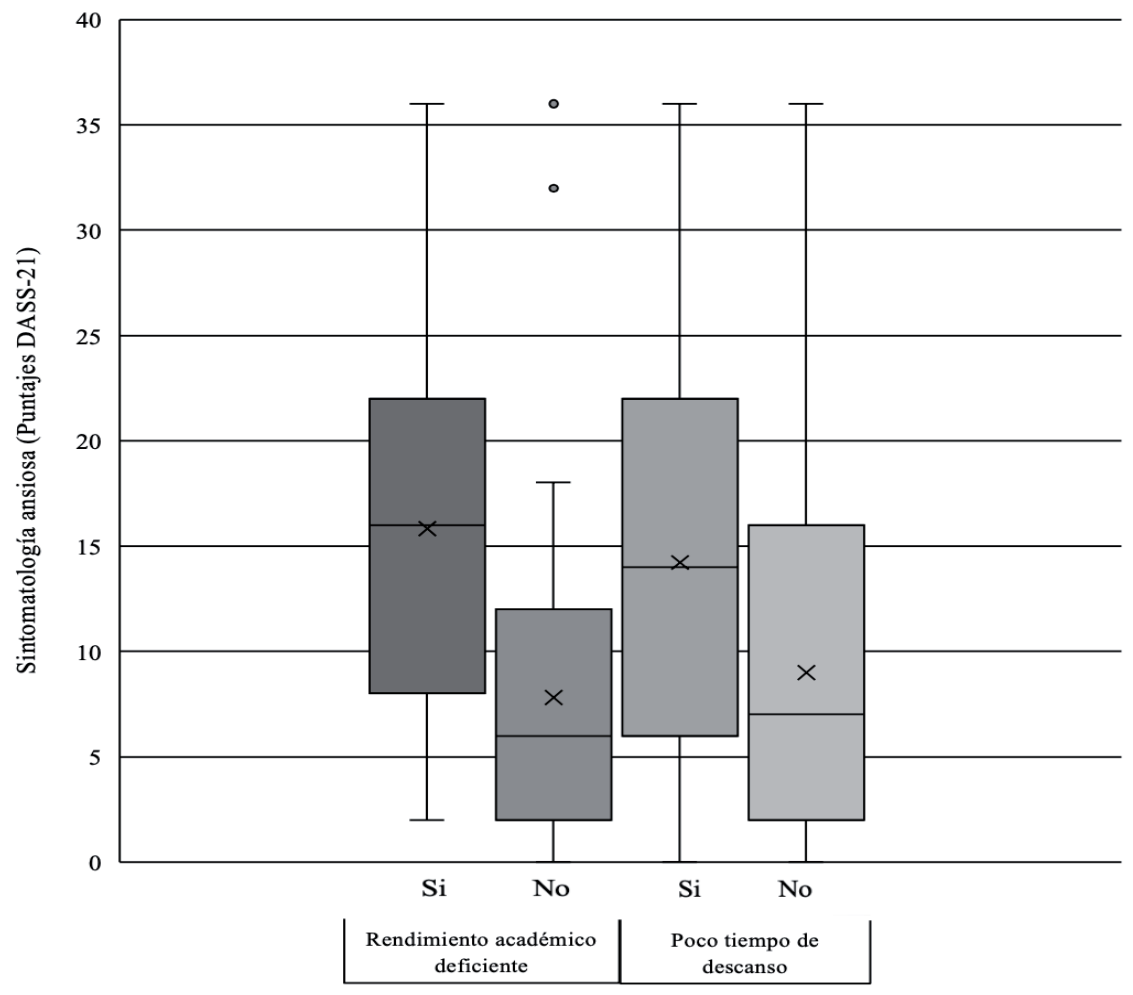

Figura 2. Variables sociodemográficas que presentan diferencias significativas respecto de la autopercepción de sintomatología ansiosa.

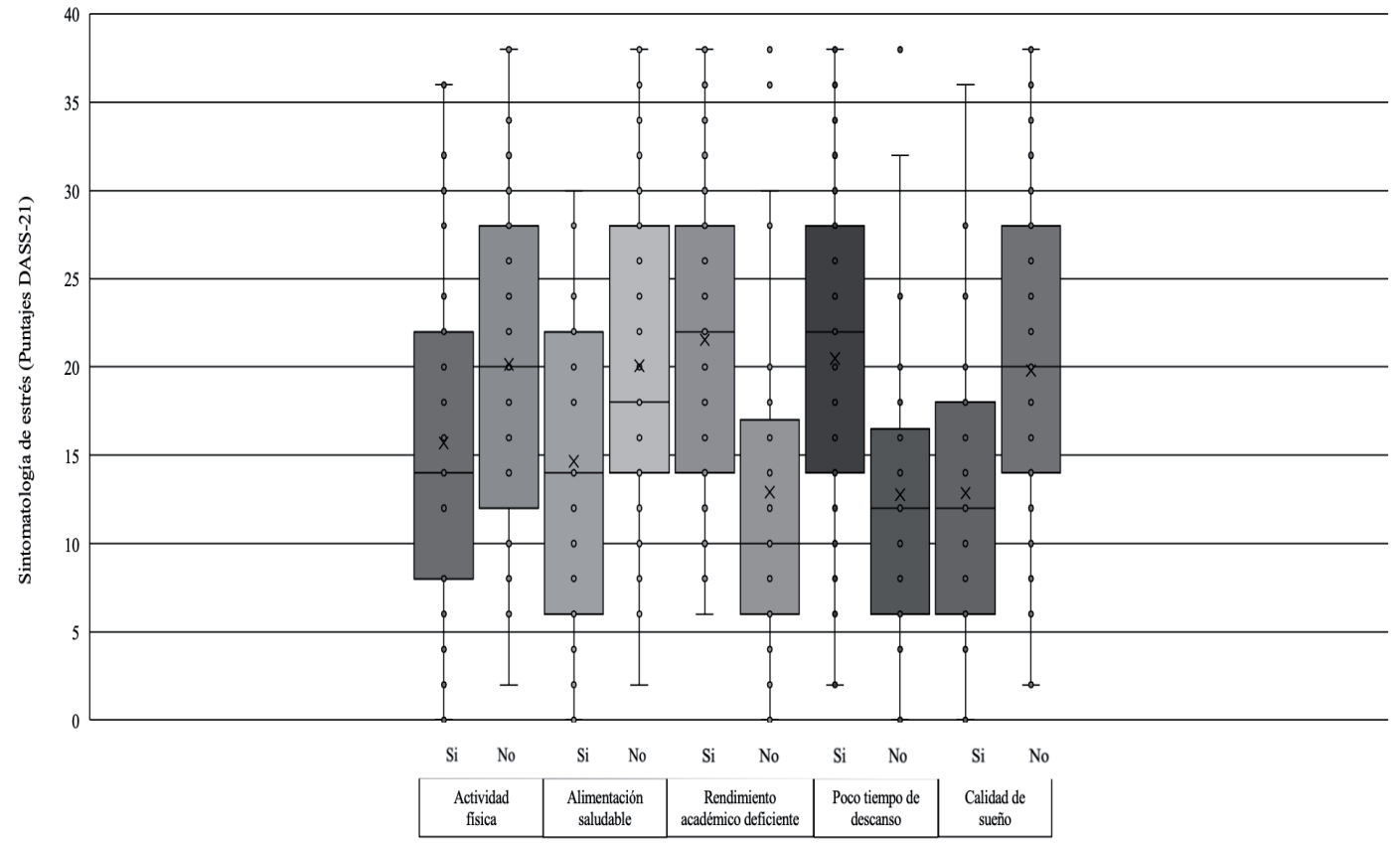

Figura 3. Variables sociodemográficas que presentan diferencias significativas respecto de la autopercepción de sintomatología de estrés. 


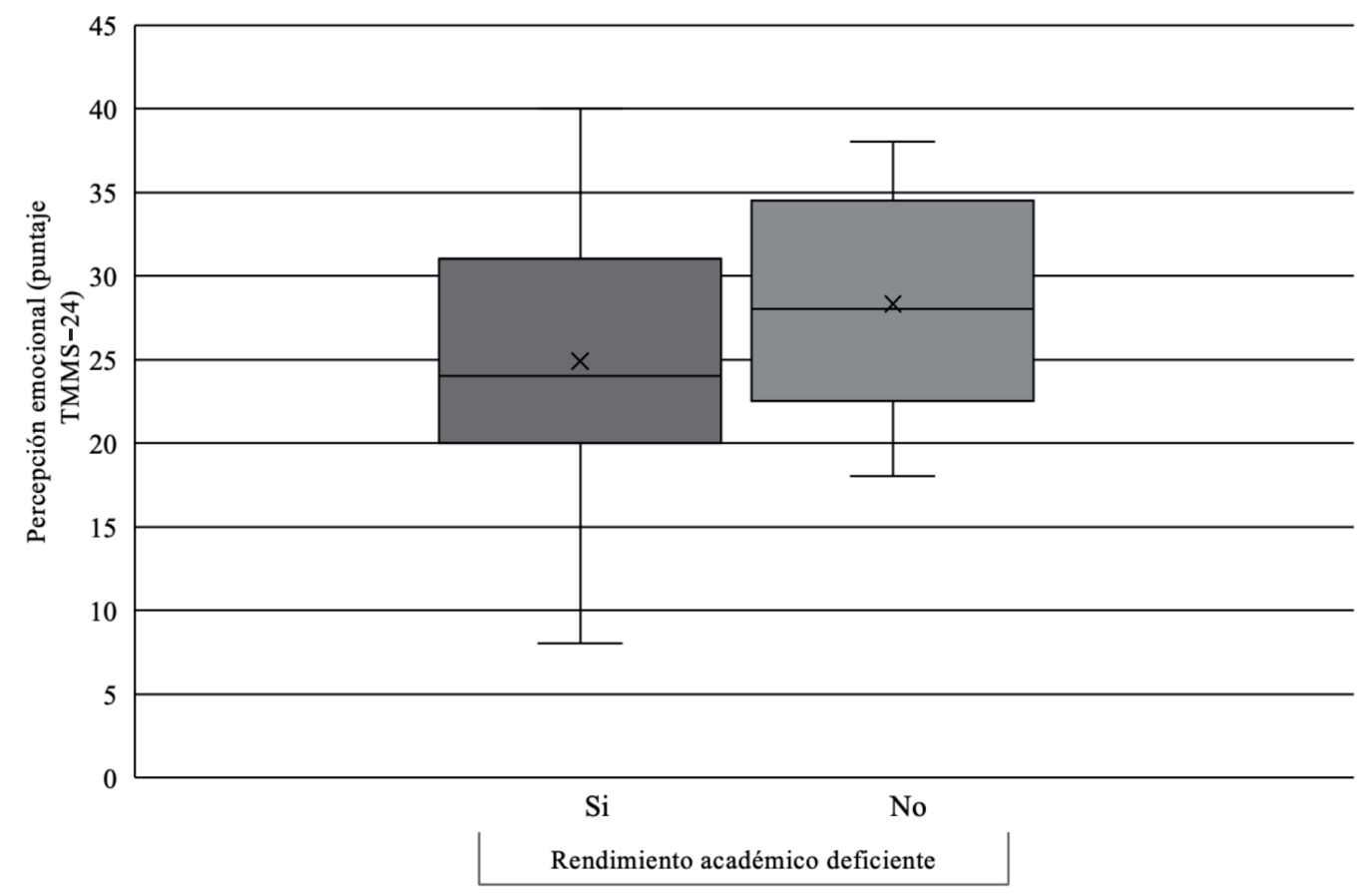

Figura 4. Variables sociodemográficas que presentan diferencias significativas respecto de la autopercepción de percepción emocional.

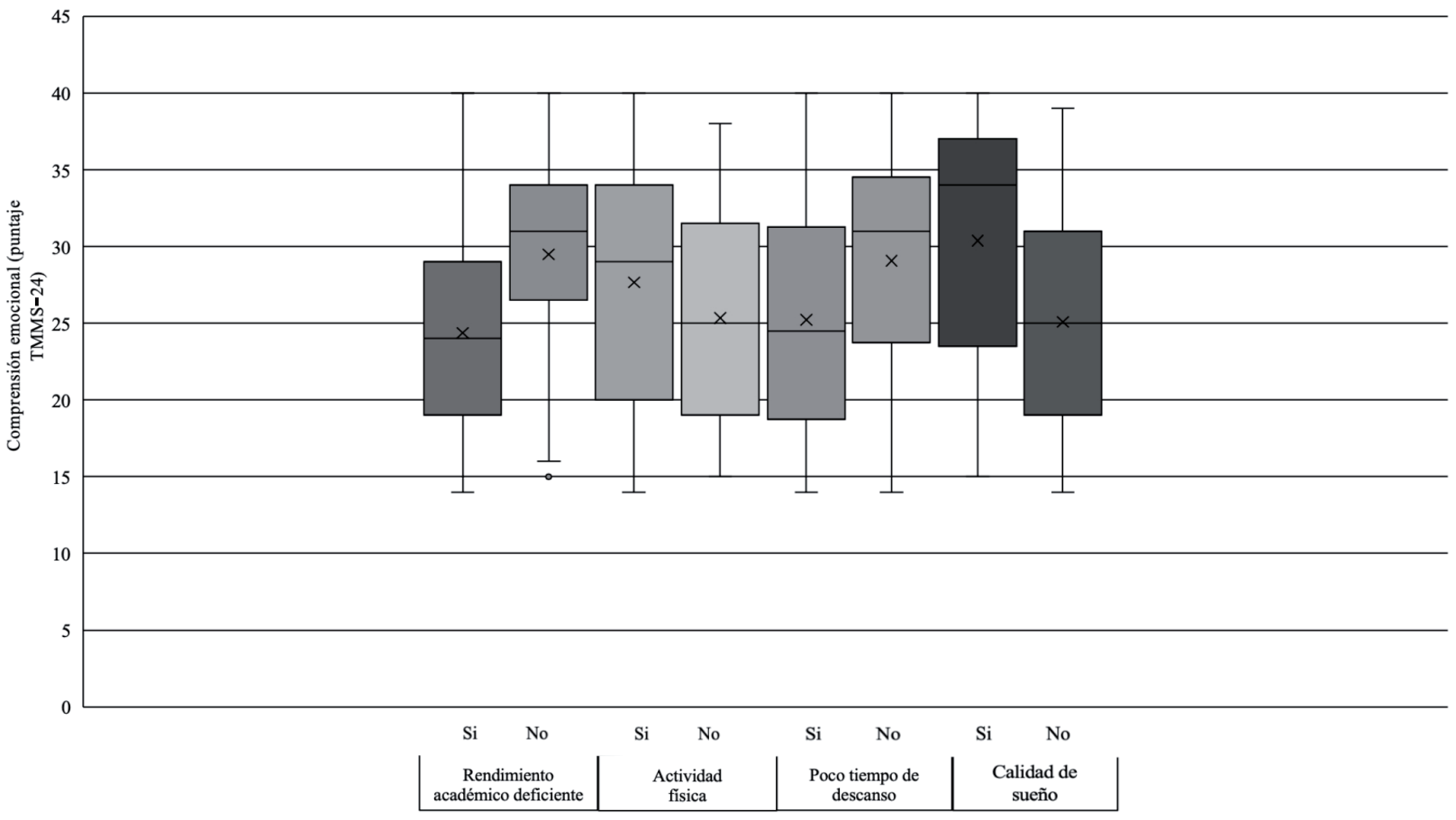

Figura 5. Variables sociodemográficas que presentan diferencias significativas respecto de la autopercepción de comprensión emocional. 
En cuanto a la variable regulación emocional, se encontraron diferencias significativas entre los grupos que presentaban buen y mal rendimiento $\left.t_{(78)}=-2,49 ; \mathrm{SE}=-4,13 ; p<0,05\right)$, entre los grupos que reportaban tener poco tiempo de descanso y adecuado tiempo de descanso $\left(t_{(78)}=4,14 ; \mathrm{SE}=7,23\right.$; $p<0,001)$, como también entre los que reportaban tener buena y mala calidad de sueńo $\left(t_{(78)}=2,94 ; \mathrm{SE}=\right.$ 5,$23 ; p<0,01$ ) (Figura 6). Las restantes variables de control no mostraron diferencias estadísticamente significativas para ninguna de las dimensiones de la inteligencia emocional.

Síntomas depresivos, ansiosos y estrés respecto a inteligencia emocional percibida. Para la variable depresión se identificó una correlación inversa, de fuerza moderada alta respecto de regulación emocional $(r=-0,66 ; p<0,01)$, además de una correlación inversa moderada de fuerza baja respecto de la autopercepción de comprensión emocional $(r=$ $-0,36 ; p<0,001)$. En cuanto a la variable síntomas ansiosos, se encontró una correlación inversa de fuerza moderada baja $(r=-0,31 ; p<0,01)$ con el autorreporte de regulación emocional.

Finalmente, para la variable estrés se encontró una correlación de tipo inversa y fuerza moderada $(r=-0,51 ; p<0,01)$ con la autopercepción de regulación emocional, además de una correlación inversa de fuerza moderada baja $(r=-0,32 ; p<0,01)$ con la autopercepción de comprensión emocional (Tabla 4).

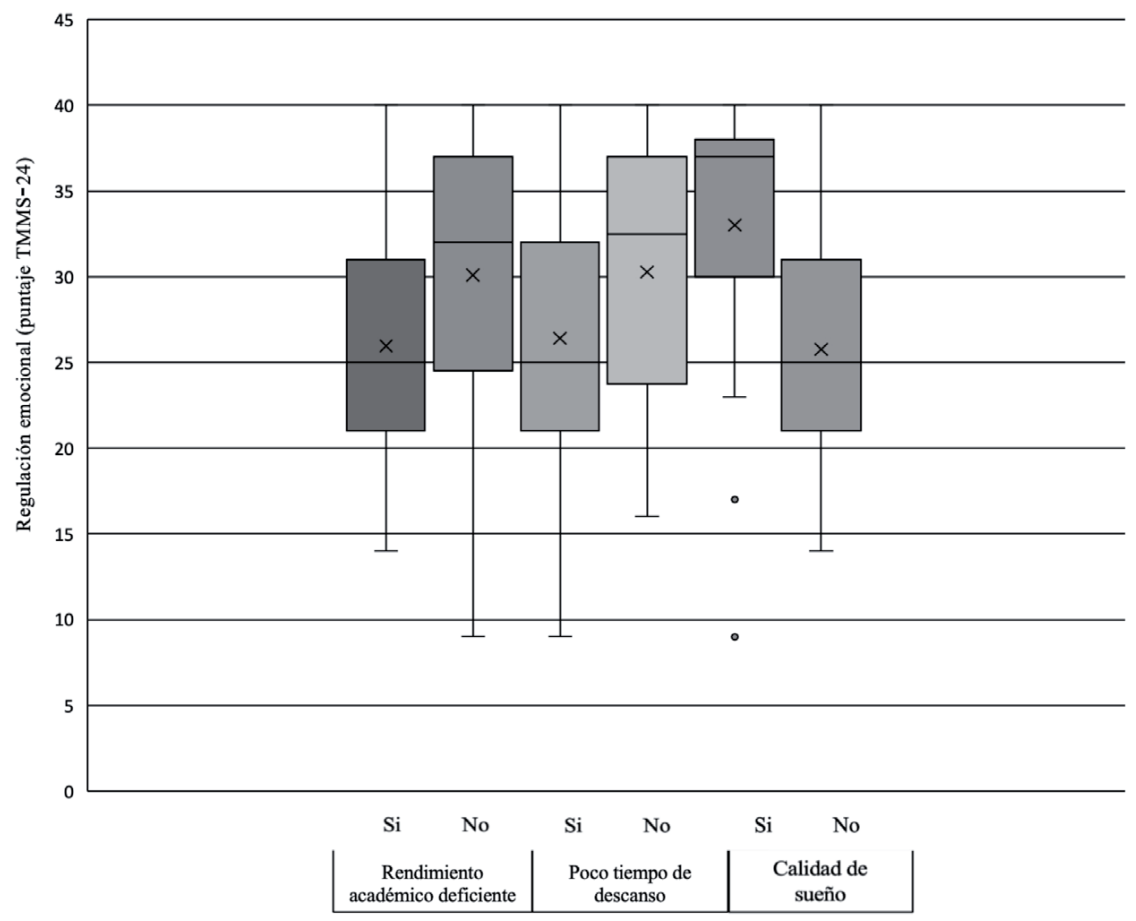

Figura 6. Variables sociodemográficas que presentan diferencias significativas respecto de la autopercepción de regulación emocional.

Tabla 4. Matriz de correlaciones entre la inteligencia emocional percibida y síntomas depresivos, ansiosos y de estrés $(n=80)$.

\begin{tabular}{|c|c|c|c|c|c|c|}
\hline & & Ansiedad & Estrés & Percepción & Comprensión & Regulación \\
\hline Depresión & 1 & $0,526^{* *}$ & $0,625^{* *}$ & $-0,03$ & $-0,358^{* *}$ & $-0,656^{* *}$ \\
\hline Ansiedad & & 1 & $0,685^{* *}$ & 0,015 & $-0,21$ & $-0,315^{* *}$ \\
\hline Estrés & & & 1 & $-0,094$ & $-0,320^{* *}$ & $-0,510^{* *}$ \\
\hline Percepción & & & & 1 & $0,328^{* *}$ & 0,209 \\
\hline Comprensión & & & & & 1 & $0,571^{* *}$ \\
\hline Regulación & & & & & & 1 \\
\hline
\end{tabular}

**La correlación es significativa al nivel 0,01 (bilateral). 


\section{DISCUSIÓN}

A partir de los resultados obtenidos, fue posible observar que hubo relación moderada a baja entre algunas de las dimensiones de la inteligencia emocional con la sintomatología evaluada. De esta forma, la regulación emocional fue la dimensión que presentó la mayor fuerza de correlación, exhibiendo relaciones inversas moderadas con cada uno de los síntomas evaluados, esto es, a mayor regulación de las emociones menor sintomatología asociada a depresión, ansiedad y estrés. Por su parte, la comprensión emocional, también presentó correlaciones inversas, pero de menor fuerza, solo con los síntomas depresivos y de estrés, lo que implicaría que una mejor comprensión de los estados emocionales se asociaría a menores síntomas depresivos y estrés en los estudiantes de enfermería de primer año.

La relación encontrada entre regulación emocional y sintomatología depresiva, ansiosa y estrés, resulta concordante con los resultados de estudios previos que evaluaron la relación de estas mismas variables en estudiantes de la salud chilenos. El trabajo de Barraza et al. ${ }^{(9)}$ encontró correlaciones inversas entre regulación emocional con los síntomas depresivos, ansiosos y estrés. En esta misma línea, el trabajo realizado por Johnson \& Blanchard $^{(29)}$ en una muestra de 287 estudiantes universitarios europeos de pregrado de distintos orígenes étnicos, encontró que la regulación emocional se relacionó de forma inversa y significativa con estrés percibido, ansiedad y depresión. Nuestros resultados, no solo van en el mismo sentido de la evidencia previa, sino que refuerzan dichos hallazgos, toda vez que presentan indicadores de correlación de mayor fuerza a los exhibidos en los estudios predecesores, al menos en esta dimensión.

La tendencia observada, tanto en los estudios previos, como en los resultados presentes, implica que la presencia de sintomatología se vincula de manera medianamente intensa con la mayor o menor habilidad para la regulación consciente de las emociones. Esto implica que una adecuada habilidad para regular las emociones, aprovecha de manera eficiente y funcional la información que acompańa a los estados emocionales, posibilitando el despliegue de una serie de estrategias que modifican los propios afectos y también los de las otras personas, permitiendo la resolución eficiente de problemas, la toma de decisiones y el crecimiento emocional e intelectual. Es por esto que la regulación emocional se considera como una habilidad de alta complejidad ${ }^{(15)}$, base para el proceso adaptativo y crucial para las/os profesionales de enfermería ${ }^{(23)}$. En este mismo orden, la evidencia neuroanatómica propuesta por Koven \& $\mathrm{Max}^{(30)}$, reconoce que los bajos niveles de regulación emocional, correlacionan con un menor volumen de materia gris en las zonas parietal frontal e inferior de la corteza, lo que es semejante a lo reportado por Marsh et al. ${ }^{(31)}$, en cuanto a que los síntomas depresivos producirían efectos de disminución del volumen de la corteza frontal de hasta un 30\%. Lo anterior reafirma la idea que un aumento de la sintomatología se vincularía de manera importante con la merma en la habilidad para regular los propios estados emocionales, factor clave en el buen desarrollo profesional y académico $^{(18,19)}$.

Por su parte, la comprensión emocional mostró correlaciones con depresión y estrés, tal como lo reportan los estudios previos ${ }^{(9,29)}$, no obstante, nuestros resultados registran correlaciones con fuerzas levemente menores a la de los estudios precedentes. A partir de lo planteado por Balluerka et al. ${ }^{(32)}$, tanto la comprensión como la regulación de emociones son factores importantes para explicar el estado de ánimo deprimido. En complemento a lo anterior, el trabajo de Ruiz -Aranda et al. ${ }^{(33)}$ propone que el estrés es un factor que aumenta de manera notable la probabilidad de padecer depresión en mujeres universitarias de carreras de salud; de esta forma el estrés puede ser considerado como un factor mediador en la percepción de inteligencia emocional.

Igualmente, resulta interesante consignar que la percepción emocional no exhibió correlaciones significativas con ninguno de los síntomas evaluados, lo cual es consistente con resultados de estudios previos ${ }^{(29)}$. En consonancia con lo anterior, el modelo tripartito de Clark y Watson ${ }^{(34)}$, propone una articulación funcional de los síntomas depresivos, ansiosos y de estrés, de esta forma el componente de afecto negativo compartido por la depresión y la ansiedad podría explicarse por los altos niveles de estrés, los cuales, si son mantenidos durante un largo periodo de tiempo, propician la reactividad del eje hipotálamo-hipófiso- 
córticosuprarrenal (eje HPA o corticotrópico), elevando los niveles de cortisol, aumentando la activación fisiológica del organismo (respuesta vagal y activación del sistema simpático) y los niveles de tolerancia al malestar emocional ${ }^{(35)}$. Lo anterior, podría explicar el hecho que la percepción emocional no haya resultado significativa en la matriz de correlación, a pesar que la muestra exhibió una alta proporción de adecuación en esta subdimensión, puesto que cuando el organismo se adapta a generar y procesar altos niveles de cortisol, va aumentando su arousal o tolerancia excitativa. Esto implica que cada vez requerirá mayor cantidad de cortisol para excitar al organismo, haciendo menos eficiente el sistema cognitivo para reconocer el estado de malestar emocional (atender a los propios estados emocionales).

Respecto a las variables de control, al compararlas con los niveles de los síntomas depresivos y de inteligencia emocional, encontramos que algunas condicionantes académicas, como el mal rendimiento académico, se vinculan a una inadecuada expresión de las dimensiones de la inteligencia emocional. Esto resulta concordante con otros estudios que previamente habían detectado que la inteligencia emocional pudiera condicionar en cierta medida el rendimiento académico $^{(36)}$ y este, a su vez, el proceso de adaptación a la universidad ${ }^{(11)}$ y el desarrollo de desórdenes emocionales ${ }^{(37)}$. Asimismo, se identificó que la mala calidad de sueño y el poco tiempo de descanso, están asociadas a deficiencias en la comprensión y regulación de las emociones propias, afectando también los niveles de estrés y ansiedad. Estos resultados concuerdan con otras investigaciones ${ }^{(38,39)}$, en donde se ha reportado que estas mismas variables influyen en la sintomatología depresiva, ansiosa y el estrés. A su vez, nuestros resultados se diferencian de lo planteado por Abdellatif ${ }^{(1)}$, que reportó que habría algunas condiciones asociadas a los hábitos y calidad del descanso que podrían estar influenciando positiva o negativamente el desempeño de las habilidades emocionales en cuestión.

Respecto de los niveles de la inteligencia emocional, en la mayoría de los casos analizados se encontraron niveles considerados adecuados, concordando con estudios recientes en estudiantes de otras ramas del área de la salud ${ }^{(9,40)}$. Respecto de los niveles de depresión, en la mayoría de los casos analizados se encontraron niveles sintomáticos altos de la patología, especialmente en la autopercepción de ansiedad y estrés. Estos niveles fueron aún más altos que los reportados en estudios similares ${ }^{(9,39)}$.

$\mathrm{Si}$ bien los resultados presentados en esta investigación resultan auspiciosos para la potencial formulación de futuras hipótesis explicativas, al ser este un estudio correlacional-no experimental, no permite identificar relaciones de causalidad entre las variables en estudio. Para analizar dichas relaciones con mayor detalle se requeriría estudiar cómo se comportan los niveles de inteligencia emocional percibida a lo largo de la carrera y/o cómo influyen estos en el surgimiento de sintomatología ansiosa, depresiva y estrés, teniendo a la vista las variables de control de tipo académicas, hábitos saludables y antecedentes de patologías psiquiátricas.

Por otra parte, dada la forma en cómo se realizó el levantamiento de los datos, no se pudo constatar que se hayan dado las condiciones óptimas para responder el cuestionario como la ausencia de otras personas interviniendo en el proceso. Tampoco se midieron parámetros asociados al proceso de responder a la encuesta, como lo son el tiempo en responder, o la identidad del estudiante. Finalmente, si bien los resultados obtenidos sugieren que podrían existir relaciones de influencia/dependencia entre estas variables, se requiere la realización de estudios subsecuentes para poder establecerlo, con muestras mejor conformadas y de mayor cuantía a fin de evitar sesgos.

\section{CONCLUSIÓN}

A partir de los resultados sobre la autopercepción de la depresión y de la inteligencia emocional, podemos plantear tres conclusiones principales.

En primer lugar, la presencia de síntomas depresivos está marcada por emociones como la angustia, temor, tristeza, preocupación y activación fisiológica involuntaria, los cuales condicionan a la persona a tener una percepción de escaso control en situaciones de alta sobrecarga emocional. Dicho estado de sobrecarga emocional, se alcanza frecuentemente en estudiantes de enfermería, dada la gran cantidad de estrés y emociones negativas que experimentan usualmente, y se vería incrementada por la competencia educativa y clínica. Dicho estado de sobrecarga es susceptible de ser regulado 
a través de la inteligencia emocional, si se cuenta con el entrenamiento adecuado. Desarrollar la inteligencia emocional en la formación académica de estudiantes de enfermería, les permitiría manejar de mejor forma las emociones negativas y las dificultades propias de los estudios universitarios en el área de la salud. Ello tendrá un impacto directo y significativo sobre el paciente que recibirá sus cuidados, ya sea en sus prácticas clínicas o después de haber egresado.

En segundo lugar, es importante cuidar las condiciones bajo las cuales se imparte la docencia universitaria, puesto que la mala calidad del sueńo y el poco tiempo de descanso son condiciones que

\section{REFERENCIAS}

1. AbdellatifSA, Hussien ESS, Hamed WE, Zoromba MA. Relation between emotional intelligence, socio-demographic and clinical characteristics of patients with depressive disorders. Arch Psychiatr Nurs [Internet]. 2017 [citado 3 jun 2020]; 31(1): 13-23. Disponible en: https://doi.org/10.1016/j. apnu.2016.07.009

2. Oriol-Granado X, Mendoza-Lira M, CovarrubiasApablaza CG, Molina-López VM. Emociones positivas, apoyo a la autonomía y rendimiento de estudiantes universitarios: el papel mediador del compromiso académico y la autoeficacia. Rev Psicodidáct. [Internet] 2017 [citado 3 jun 2020]; 22(1): 45-53. Disponible en: https://doi. org/10.1016/S1136-1034(17)30043-6

3. Blanco H, Aguirre JF, Barrón JC, Blanco JR. Composición factorial de la Escala de Autoeficacia Académica en universitarios mexicanos. Form Univ [Internet]. 2016 [citado 3 jun 2020]; 9(2): 81-88. Disponible en: http://dx.doi.org/10.4067/ S0718-50062016000200009

4. Antúnez Z, Vinet EV. Problemas de salud mental en estudiantes de una universidad regional chilena. Rev méd Chile [Internet]. 2013 Feb [citado 22 nov 2018]; 141(2): 209-16. Disponible en: https://scielo.conicyt.cl/scielo.php?script=sci_ abstract\&pid=S003498872013000200010\&lng= es\&nrm=iso\&tlng=pt.

5. Aradilla-Herrero A, Tomás-Sábado J, GómezBenito J. Associations between emotional intelligence, depression and suicide risk in nursing students. Nurse Educ Today [Internet]. 2014 [citado 3 jun 2020]; 34(4): 520-525. Disponible afectan aspectos emocionales de los estudiantes, $y$ repercuten negativamente en su salud y rendimiento académico. Rediseńar estos espacios es una responsabilidad prioritaria para los docentes de enfermería.

En tercer lugar, la alta incidencia de sintomatología ansiosa y estrés en estudiantes de primer año de enfermería debe ser el foco de nuevas políticas universitarias destinadas a mejorar las condiciones de los estudiantes. Dichas políticas deben ser diseñadas en conjunto con las Universidades, el cuerpo docente, y los alumnos, para acordar mecanismos concretos que mejoren la salud mental del alumnado.

en: https://doi.org/10.1016/j.nedt.2013.07.001

6. Sánchez IDR, Acedo MAD, Herrera SS, García MLB. La inteligencia emocional en estudiantes universitarios: diferencias entre el grado de maestro en educación primaria y los grados en ciencias. Int J Dev Educ Psychol [Internet]. 2016 [citado 25 oct 2017]; 2(1): 51-61. Disponible en: https://doi. org/10.17060/ijodaep.2016.n1.v2.176

7. Cilar L, Barr O, Štiglic G, Pajnkihar M. Mental well-being among nursing students in Slovenia and Northern Ireland: A survey. Nurse Educ Pract [Internet]. 2019 [citado 3 jun 2020]; 39:130-5. Disponible en: https://linkinghub.elsevier.com/ retrieve/pii/S1471595318305365

8. Von-Bischhoffshausen-P K, Wallem-H A, Allendes-A A, Díaz-M R. Prevalencia de Bruxismo y Estrés en Estudiantes de Odontología de la Pontificia Universidad Católica de Chile. Int J odontostomat [Internet]. 2019 Mar [citado 3 jun 2020]; 13(1): 97-102. Disponible en: https://scielo.conicyt.cl/scielo.php?script $=$ sci abstract\&pid=S0718-381X2019000100097\&lng= es\&nrm=iso\&tlng=es.

9. Barraza-López RJ, Muñoz-Navarro NA, BehrensPérez CC. Relación entre inteligencia emocional y depresión-ansiedad y estrés en estudiantes de medicina de primer año. Rev chil neuropsiquiatr [Internet]. 2017 abril [citado 14 ene 2019]; 55(1): 18-25. Disponible en: http://www. scielo.cl/scielo.php?script=sci_arttext\&pid=S071792272017000100003\&lng=en\&nrm=iso\&tlng= en.

10. Orak RJ, Farahani MA, Kelishami FG, Seyedfatemi N, Banihashemi S, Havaei F. Investigating the effect of emotional intelligence education 
on baccalaureate nursing students' emotional intelligence scores. Nurse Educ Pract [Internet]. 2016 [citado 14 ene 2019]; 20: 64-69. Disponible en: https://doi.org/10.1016/j.nepr.2016.05.007

11. Garg R, Levin E, Tremblay L. Emotional intelligence: impact on post-secondary academic achievement. Soc Psychol Educ [Internet]. 2016 [citado 14 ene 2019]; 19(3): 627-642. Disponible en: https://doi.org/10.1007/s11218-016-9338-x

12. Berrocal PF, Pacheco NE. La Inteligencia Emocional y la educación de las emociones desde el Modelo de Mayer y Salovey. Revista Interuniversitaria de Formación del Profesorado [Internet]. 2005 [citado 14 ene 2019]; 19(3): 63-93. Disponible en: http://www.redalyc.org/articulo. oa? id=27411927005

13. Raghubir AE. Emotional intelligence in professional nursing practice: A concept review using Rodgers's evolutionary analysis approach. Int J Nurs Sci [Internet]. 2018 [citado 14 ene 2019]; 5(2): 126-130. Disponible en: https://doi. org/10.1016/j.ijnss.2018.03.004

14. Treglown L, Furnham A. Birds of a feather work together: The role of emotional intelligence and cognitive ability in workplace interaction and advice networks. Personality and Individual Differences [Internet]. 2020 May [citado 3 jun 2020]; 158: 109833. Disponible en: https://linkinghub. elsevier.com/retrieve/pii/S0191886920300234

15. Gómez-Romero MJ, Limonero JT, Trallero JT, Montes-Hidalgo J, Tomás-Sábado J. Relación entre inteligencia emocional, afecto negativo y riesgo suicida en jóvenes universitarios. Ansiedad Estrés [Internet]. 2018 [citado 3 jun 2020]; 24(1): 18-23. https://doi.org/10.1016/j.anyes.2017.10.007

16. Suárez Y, Cervantes DR, Domínguez CC. Ideación suicida y su relación con la inteligencia emocional en universitarios colombianos. Revista Salud Uis [Internet]. 2016 [citado 3 jun 2020]; 48(4): 470-8. Disponible en: https://dialnet.unirioja.es/servlet/ articulo? codigo $=6752733$

17. Birks Y, McKendree J, Watt I. Emotional intelligence and perceived stress in healthcare students: a multi-institutional, multi-professional survey. BMC Medical Education [Internet]. 2009 Sep [citado 25 oct 2017]; 9: 61. Disponible en: https://doi.org/10.1186/1472-6920-9-61

18. Hutchinson M, Hurley J, Kozlowski D, Whitehair L. The use of emotional intelligence capabilities in clinical reasoning and decision-making: A qualitative, exploratory study. J Clin Nurs John Wiley Sons Inc [Internet]. 2018 Feb [citado 3 jun 2020]; 27(3-4): e600-10. Disponible en: https:// doi.org/10.1111/jocn.14106

19. Arora S, Russ S, Petrides KV, Sirimanna P,
Aggarwal R, Darzi A, et al. Emotional intelligence and stress in medical students performing surgical tasks. Acad Med [Internet]. 2011 [citado 25 oct 2017]; 86(10): 1311-1317. Disponible en: 10.1097/ ACM.0b013e31822bd7aa

20. Perestelo-Pérez L. Standards on how to develop and report systematic reviews in Psychology and Health. Int J Clin Health Psychol [Internet]. 2013 [citado 25 oct 2017]; 13(1): 49-57. Disponible en: https://doi.org/10.1016/S1697-2600(13)70007-3

21. Sharon D, Grinberg K. Does the level of emotional intelligence affect the degree of success in nursing studies? Nurse Educ Today [Internet]. 2018 [citado 3 jun 2020]; 64: 21-26. Disponible en: https://doi. org/10.1016/j.nedt.2018.01.030

22. Parnell RB, Onge JLS. Teaching safety in nursing practice: Is emotional intelligence a vital component? Teach Learn Nurs [Internet]. 2015 [citado 25 oct 2017]; 10(2): 88-92. Disponible en: https://doi.org/10.1016/j.teln.2014.11.001

23. Allen DE, Ploeg J, Kaasalainen S. The relationship between emotional intelligence and clinical teaching effectiveness in nursing faculty. J Prof Nurs [Internet]. 2012 [citado 25 oct 2017]; 28(4): 231-240. Disponible en: https://doi.org/10.1016/j. profnurs.2011.11.018

24. Schoeps K, De la Barrera U, Montoya-Castilla I. Impact of emotional development intervention program on subjective well-being of university students. High Educ [Internet]. 2019 [citado 3 jun 2020]; 1-19. Disponible en: https://doi. org/10.1007/s10734-019-00433-0

25. Kousha M, Bagheri HA, Heydarzadeh A. Emotional intelligence and anxiety, stress, and depression in Iranian resident physicians. J Fam Med Prim Care [Internet]. 2018 [citado 3 jun 2020]; 7(2): 420. Disponible en: 10.4103/jfmpc. jfmpc_154_17

26. Espinoza-Venegas M, Sanhueza-Alvarado O, Ramírez-Elizondo N, Sáez-Carrillo K. Validação do construto e da confiabilidade de uma escala de inteligência emocional aplicada a estudantes de enfermagem. Rev Latino-Am Enfermagem [Internet]. 2015 Feb [citado 2 feb 2018]; 23(1): 139-47. Disponible en: http://www.scielo.br/ scielo.php?script $=$ sci_abstract $\&$ pid $=$ S0104$11692015000100139 \& \operatorname{lng}=$ pt\&nrm=iso\&tlng=en

27. Antúnez Z, Vinet EV. Escalas de depresión, ansiedad y Estrés (DASS-21): Validación de la Versión abreviada en Estudiantes Universitarios Chilenos. Ter Psicológica [Internet]. 2012 [citado 25 oct 2017]; 30(3): 49-55. Disponible en: http:// dx.doi.org/10.4067/S0718-48082012000300005

28. Cheung T, Wong SY, Wong KY, Law LY, Ng $\mathrm{K}$, Tong MT, et al. Depression, Anxiety and 
Symptoms of Stress among Baccalaureate Nursing Students in Hong Kong: A Cross-Sectional Study. Int J Environ Res Public Health [Internet]. 2016 Ago [citado 17 nov 2018]; 13(8): 779. Disponible en: https://www.mdpi.com/1660-4601/13/8/779 .

29. Johnson SK, Blanchard A. Emotional intelligence and mental health: Stress and symptom reporting pathways. J Ment Health Couns [Internet]. 2016 [citado 25 oct 2017]; 38(1): 79-92. Disponible en: https://doi.org/10.17744/mehc.38.1.06

30. Koven NS, Max LK. Basal salivary oxytocin level predicts extra-but not intra-personal dimensions of emotional intelligence. Psychoneuroendocrinology [Internet]. 2014 [citado 25 oct 2017]; 44: 2029. Disponible en: https://doi.org/10.1016/j. psyneuen.2014.02.018

31. Marsh R, Gerber AJ, Peterson BS. Neuroimaging studies of normal brain development and their relevance for understanding childhood neuropsychiatric disorders. J Am Acad Child Adolesc Psychiatry [Internet]. 2008 [citado 3 jun 2020]; 47(11): 1233-1251. Disponible en: https:// doi.org/10.1097/CHI.0b013e318185e703

32. Balluerka N, Aritzeta A, Gorostiaga A, Gartzia L, Soroa G. Emotional intelligence and depressed mood in adolescence: A multilevel approach. Int J Clin Health Psychol [Internet]. 2013 [citado 3 jun 2020]; 13(2): 110-117. Disponible en: https://doi. org/10.1016/S1697-2600(13)70014-0

33. Ruiz-Aranda D, Extremera N, Pineda-Galan C. Emotional intelligence, life satisfaction and subjective happiness in female student health professionals: the mediating effect of perceived stress. J Psychiatr Ment Health Nurs [Internet]. 2014 [citado 25 oct 2017]; 21(2): 106-113. Disponible en: https://doi.org/10.1111/jpm.12052

34. Clark LA, Watson D. Tripartite model of anxiety and depression: psychometric evidence and taxonomic implications. J Abnorm Psychol [Internet]. 1991 [citado 25 oct 2017]; 100(3): 316. Disponible en: http://news.schoolsdo.org/wp- content/uploads/2016/09/tripartite-model-1991. pdf

35. Sánchez-Pedreño FO. Lecciones de Psiquiatría [Internet]. 2010 [citado 12 jun 2020]. Editorial Médica Panamericana. 650 p. Disponible en: https://www.medicapanamericana.com/Libros/ Libro/3879/Lecciones-de-Psiquiatria.html

36. Costa A, Faria L. Implicit Theories of Emotional Intelligence, Ability and Trait-Emotional Intelligence and Academic Achievement. Psihol Teme [Internet]. 2020 [citado 12 jun 2020]; 29(1): 43-61. Disponible en: https://doi.org/10.31820/ pt.29.1.3

37. Yanni JR. Academic achievement \& emotional disturbance: Examining the use of growth scale values to measure academic achievement of students with emotional disturbance $[\mathrm{PhD}$ Thesis. Internet]. Fairleigh Dickinson University; 2016 [citado 12 jun 2020]. Disponible en: https:// search.proquest.com/openview/9a02e15a49040d0 0f4080cdd7c526950/1?pq-origsite $=$ gscholar $\& \mathrm{cbl}=$ 18750\&diss $=\mathrm{y}$

38. Cheung T, Wong SY, Wong KY, Law LY, Ng $\mathrm{K}$, Tong MT, et al. Depression, anxiety and symptoms of stress among baccalaureate nursing students in Hong Kong: a cross-sectional study. Int J Environ Res Public Health [Internet]. 2016 [citado 2 feb 2018]; 13(8): 779. Disponible en: https://doi.org/10.3390/ijerph13080779

39. Vergara KA, Cárdenas SD, Martínez FG. Síntomas de depresión, ansiedad y estrés en estudiantes de odontología: prevalencia y factores relacionados. Rev Colomb Psiquiatr [Internet]. 2013 [citado 2 feb 2018]; 42(2): 173-181. Disponible en: https:// doi.org/10.1016/S0034-7450(13)70004-0

40. Birks Y, McKendree J, Watt I. Emotional intelligence and perceived stress in healthcare students: a multi-institutional, multi-professional survey. BMC Med Educ [Internet]. 2009 [citado 2 feb 2018]; 9(1): 61. Disponible en: https://doi. org/10.1186/1472-6920-9-61 